\title{
Conditioning reservoir models on rate data using ensemble smoothers
}

\author{
Geir Evensen $^{1,2}$ (D) $\cdot$ Kjersti Solberg Eikrem ${ }^{1}$
}

Received: 21 June 2017 / Accepted: 30 April 2018 / Published online: 22 June 2018

(c) The Author(s) 2018

\begin{abstract}
There are several issues to consider when we use ensemble smoothers to condition reservoir models on rate data. The values in a time series of rate data contain redundant information that may lead to poorly conditioned inversions and thereby influence the stability of the numerical computation of the update. A time series of rate data typically has correlated measurement errors in time, and negligence of the correlations leads to a too strong impact from conditioning on the rate data and possible ensemble collapse. The total number of rate data included in the smoother update will typically exceed the ensemble size, and special care needs to be taken to ensure numerically stable results. We force the reservoir model with production rate data derived from the observed production, and the further conditioning on the same rate data implies that we use the data twice. This paper discusses strategies for conditioning reservoir models on rate data using ensemble smoothers. In particular, a significant redundancy in the rate data makes it possible to subsample the rate data. The alternative to subsampling is to model the unknown measurement error correlations and specify the full measurement error covariance matrix. We demonstrate the proposed strategies using different ensemble smoothers with the Norne full-field reservoir model.
\end{abstract}

Keywords History matching $\cdot$ Ensemble smoothers $\cdot$ Iterative ensemble smoothers $\cdot$ Production data

\section{Introduction}

Ensemble methods for data assimilation and parameter estimation (see, e.g., $[9,12,13])$ are now well established in the reservoir-engineering community for history matching reservoir models. Following the first application of Ensemble Kalman Filter (EnKF) with reservoir simulation models by [25], there is now a large number of publications that address the estimation of parameters in reservoir simulation models using EnKF, and we refer to the review by [1] and references therein for an overview.

Skjervheim et al. [28] introduced the use of Ensemble Smoother (ES) as an alternative to the sequential EnKF for history matching reservoir models. ES differs from EnKF by computing a global update in the space-time domain

\footnotetext{
Geir Evensen

geir.evensen@norceresearch.no

Kjersti Solberg Eikrem

kjersti.solberg.eikrem@norceresearch.no

1 International Research Institute of Stavanger, Bergen, Norway

2 Nansen Environmental and Remote Sensing Center, Bergen, Norway
}

rather than using recursive updates in time as in EnKF. Thus, we avoid the sequential updating of the realizations with associated restarts. ES is otherwise similar to EnKF by using an ensemble of realizations to represent the uncertainty and a variance-minimizing update scheme.

Van Leeuwen and Evensen [21] initially proposed ES and showed that EnKF and ES both solve the same Bayesian formulation. EnKF uses a recursion in time under the assumption of measurements that are independent in time and a Markov reservoir model, where the model solution at a time step is only dependent on the model solution at the previous time step. For linear dynamical models and measurements, EnKF and ES provide the same solutions as is shown by [11]. [21] found that EnKF delivers results superior to ES in an application with an ocean circulation model. [15] later explained that for nonlinear dynamical models, and in particular models with chaotic dynamics, EnKF is superior to ES since the recursive updates keep the model on track and close to the true solution represented by the measurements. Thus, further research on ES in other applications has been slow, and ES has not received much attention in the data-assimilation community.

Despite previous somewhat disappointing results with ES, [28] tried the ES again with a reservoir model based on 
the knowledge that we can characterize reservoir models as weakly nonlinear, and not chaotic. Thus, reservoir models should be more suitable to be used with ES than the highly nonlinear models previously used to examine ES. The ground-breaking result from [28] was that similar performances and quality of results were obtained using ES and EnKF in a real reservoir test case. This finding has triggered a fast development of new smoother formulations and applications of smoothers, and ensemble smoothers are now the preferred choice in the petroleum community.

There are some significant differences when using ensemble smoothers versus EnKF in history matching. EnKF considers a mixed state- and parameter-estimation problem where both the parameters and dynamical state variables are updated recursively in time. In ES, the whole ensemble of model realizations is simulated forward in time, once, to generate a prediction, then the ensemble of parameters is updated, and finally, the ensemble is rerun using the updated parameters to create the final history-matched ensemble of model predictions. Thus, the ensemble smoothers consider a standard parameterestimation problem.

There are some practical advantages of using ensemble smoothers instead of EnKF.

- There is no sequential updating and associated stopping and restarting of the ensemble of reservoir models, which adds a lot of computation time in the EnKF.

- The dynamical model variables are never updated, and one removes the possibility of creating unphysical model solutions that may cause problems for the simulator.

- There is no need to implicitly assume that data are independent in time as in EnKF, and proper timecorrelated measurement errors can be modeled and taken into account when computing the update.

- The standard parameter-estimation problem is also much easier to grasp by reservoir engineers since it is very similar to the concept used in most other software developed for history matching.

However, the main reason for introducing ES as a historymatching method was the simplification of the whole process where it became possible to update much more general parameters than in EnKF. In EnKF it is required that all realizations have the same grid and active grid cells, and mainly petrophysical parameters such as gridcell porosity and permeability, and scalar parameters such as fault transmissibilities, are updated in addition to the state variables. When using ensemble smoothers, it is possible to update so-called hyperparameters such as channel facies directions, sub-seismic fault density, and even structural parameters including the grid itself [16, 27, 29]. Hyperparameters are parameters that are input to the geological modeling process in contrast to the parameters that are output from the geological modeling and which we usually update in the conditioning.

Following the introduction of ES for history matching by [28], two iterative variants of the smoother formulation were introduced, i.e., ES with Multiple Data Assimilation (ESMDA) by [8] and Iterative ES (IES) by [4, 5]. They both found that iterations of the smoother update resolved some issues with nonlinearity and gave better results than what was obtained by ES. There is now a range of new smoother developments and applications based on the original iterative variants, e.g., [18-20, 22, 23].

Although there are many benefits of replacing EnKF with ensemble smoothers, the smoothers also introduce some new challenges.

- The number of measurements used in the computation of the update may become very large since all data are conditioned on simultaneously, whereas in EnKF they are processed recursively in time. A large measurement vector compared to the number of ensemble members implies that the inversion is of low rank and we must take special care when computing the updated ensemble.

- The assumption of time-independent errors in rate measurements is not valid, even though EnKF implicitly imposes this assumption when conditioning on rate data. The implicit use of this assumption is one of the reasons one has often seen examples of filter divergence when using EnKF with reservoir models and there is no difference when using the ensemble smoothers [20]. In ensemble smoothers, it is possible to take the time correlation in measurement errors into account. However, the inclusion of the time correlations leads to a non-diagonal measurement error covariance matrix that needs to be inverted, and this inversion poses a computational problem when using many observations.

- The final issue relates to the conditioning on redundant data or information. To illustrate, if we go from monthly to weekly rate data we quadruple the number of measurements but do we also quadruple the amount of information we use in the conditioning? The conditioning on redundant information leads to a numerically ill-conditioned inversion in the smoother, while in the EnKF the recursive processing of measurements often resolves this computational issue by recursively inverting small and numerically wellconditioned matrices.

Only a few publications have previously considered the impact of correlated measurement errors and dependent measurement information in data assimilation and we refer to [3], who proposed a scheme for optimizing information content when collecting measurements, [30] who examined the impact of neglecting measurement error correlations on the data-assimilation estimate, and [24] who considered 
the information contained in measurements with varying degrees of redundancy and measurement-error correlations.

Eikrem et al. [7] compared the effect of using different uncertainty estimates for inverted seismic data when history matching a reservoir model, and observed that in some cases the history matching results were improved when including the correlations in the error in the seismic data.

In time series of oil, gas, and water rate data, there will be redundant information contained in the data since the data has high resolution in time compared to the time scales of the internal dynamical variability of the reservoir models. Also, the allocation procedures used to obtain the rate data introduces error correlations in time.

The conditioning on dependent and redundant rate data introduces new potential pitfalls when using ensemble methods compared to previous typical minimization algorithms. Traditionally, we formulated the history-matching problem as the minimization of a cost function that measures the difference between observed and predicted rates computed given a set of uncertain parameters [2, 26]. Typically, one would also include a term constraining the deviation from the prior estimate of the parameters. In this formulation, it is not crucial to include time correlations and avoid redundancy in the rate data, since adding more data does not necessarily lead to a stronger update. It is the relative weight of the prior and measurement terms that determine the strength of the update. When introducing EnKF and ensemble smoothers for solving the history-matching problem, we have continued to use the rate data as done traditionally. We have not considered the additional impact of the data from recursively introducing the same information again and again when we update the ensemble. The result has been several studies where the estimated uncertainty is too low, and we have seen examples of "ensemble collapse" [1].

In this paper, we show that a subsampling of the rate data in the conditioning can be beneficial since it resolves the challenges discussed above. We take a radical approach for demonstration purposes, where we condition only on the total accumulated production of oil, gas, and water at the final time for each well. We base this approach on the assumption that there is a redundancy in a time series of rate data, and that the total accumulated production contains a major part of the information in a time series of rate data. The advantages of strongly subsampling the rate data are several. By avoiding the conditioning on rate data located closely in time, an appropriate subsampling of the rate data will reduce the conditioning on redundant information in the data. By only conditioning on the total accumulated production, we do not have to consider the time correlations of the measurement errors. Thus, we can consistently use a diagonal measurement error covariance matrix, as long as there are no significant error correlations between different wells (such relationships may exist in some cases, but we do not consider them here). The conditioning on a low number of measurements which is less than the number of ensemble members, simplify the numerical computation of the update since the inverted matrix may be of full rank and the dimension of the ensemble space is large enough to represent the information provided by the measurements. Finally, we avoid the conditioning on rate data that have already been used to derive the produced reservoir fluid volumes used to force the reservoir model for the historical period.

We start by giving the update equations used in ES, followed by a discussion on the impact of conditioning on measurements with redundant information as well as the effect of including or excluding correlated measurement errors. Then we discuss properties of time series of production rate data before we present an application where we use the Norne field model.

\section{Ensemble update equations}

Evensen [10] formulates the EnKF analysis scheme as a function of the ensemble without reference to the ensemble covariance matrix, which allows for an efficient numerical implementation as well as an alternative interpretation of the method. We follow the slightly updated formulation from [12].

For the history-matching problem, the state vector $\mathbf{x}$ contains all the uncertain parameters and also augments the predicted measurements, which represents a simulation of the model to produce the observed well rates. The measurement operator $\mathbf{H}$ is then a linear operator that selects the predicted observations from the ensemble (or corresponding rows and columns in the covariance matrix).

We define the matrix $\mathbf{A}$, whose columns are the ensemble members $\mathbf{x}_{i} \in \mathfrak{R}^{n}$ and the matrix $\mathbf{D}$ whose columns are the perturbed measurements $\mathbf{d}_{i}=\overline{\mathbf{d}}+\mathbf{e}_{i} \in \mathfrak{R}^{m}$, by

$\mathbf{A}=\left(\mathbf{x}_{1}, \mathbf{x}_{2}, \ldots, \mathbf{x}_{N}\right) \in \mathfrak{R}^{n \times N}$,
$\mathbf{D}=\left(\mathbf{d}_{1}, \mathbf{d}_{2}, \ldots, \mathbf{d}_{N}\right) \in \mathfrak{R}^{m \times N}$,
$\mathbf{E}=\left(\mathbf{e}_{1}, \mathbf{e}_{2}, \ldots, \mathbf{e}_{N}\right) \in \mathfrak{R}^{m \times N}$,

where $N$ is the number of ensemble members, $n$ is the size of the model state vector and $m$ is the number of measurements.

We can then define the ensemble covariances by

$$
\begin{aligned}
\mathbf{P}^{\mathrm{e}} & =\frac{1}{N-1} \mathbf{A}^{\prime}\left(\mathbf{A}^{\prime}\right)^{\mathrm{T}} \in \mathfrak{R}^{n \times n}, \\
\mathbf{R}^{\mathrm{e}} & =\frac{1}{N-1} \mathbf{E} \mathbf{E}^{\mathrm{T}} \in \mathfrak{R}^{m \times m},
\end{aligned}
$$

where the prime denotes the ensemble of perturbations with the ensemble mean subtracted. 
The ensemble-analysis equation, expressed in terms of the ensemble matrices, is

$$
\mathbf{A}^{\mathrm{a}}=\mathbf{A}+\mathbf{P}^{\mathrm{e}} \mathbf{H}^{\mathrm{T}}\left(\mathbf{H} \mathbf{P}^{\mathrm{e}} \mathbf{H}^{\mathrm{T}}+\mathbf{R}^{\mathrm{e}}\right)^{-1}(\mathbf{D}-\mathbf{H A}),
$$

where we have skipped the superscript, $\mathrm{f}$, on $\mathbf{A}$ and $\mathbf{P}^{\mathrm{e}}$. Using the definitions of the ensemble error covariance matrices in Eqs. (4) and (5), the analysis can be expressed as

$$
\mathbf{A}^{\mathrm{a}}=\mathbf{A}+\mathbf{A}^{\prime} \mathbf{A}^{\prime \mathrm{T}} \mathbf{H}^{\mathrm{T}}\left(\mathbf{H} \mathbf{A}^{\prime}\left(\mathbf{A}^{\prime}\right)^{\mathrm{T}} \mathbf{H}^{\mathrm{T}}+\mathbf{E E}^{\mathrm{T}}\right)^{-1}(\mathbf{D}-\mathbf{H A}),
$$

where all references to the error covariance matrices are replaced with their ensemble representations.

It was shown in [10] that the analysis Eq. (7) can be written as

$\mathbf{A}^{\mathrm{a}}=\mathbf{A} \mathbf{X}$,

with the transition matrix $\mathbf{X} \in \Re^{N \times N}$ defined as

$\mathbf{X}=\mathbf{I}+\mathbf{S}^{\mathrm{T}} \mathbf{C}^{-1}(\mathbf{D}-\mathbf{H A})$.

We have defined the matrix $\mathbf{S} \in \Re^{m \times N}$ holding the measurements of the ensemble perturbations by

$\mathbf{S}=\mathbf{H A}^{\prime}$

and the matrix $\mathbf{C} \in \Re^{m \times m}$,

$$
\begin{aligned}
\mathbf{C} & =\mathbf{S S}^{\mathrm{T}}+\mathbf{E E}^{\mathrm{T}} \\
& =\mathbf{S S}^{\mathrm{T}}+(N-1) \mathbf{R}^{\mathrm{e}} \\
& \approx \mathbf{S S}^{\mathrm{T}}+(N-1) \mathbf{R} .
\end{aligned}
$$

In the definition of $\mathbf{C}$, we have represented measurement errors both using the ensemble representation (5) and an analytically prescribed error covariance matrix R. For computational reasons, it is an advantage to express the measurement error covariance matrix by an ensemble of measurement perturbations. The perturbations are usually more straightforward to sample than explicit modeling of the off-diagonal terms in R. (Chap. 14 [12]) developed an algorithm that can compute this inversion for cases with a vast number of measurements containing redundant information as well as for observations with correlated errors. To summarize, we compute the updated ensemble from Eq. (8) and only need to evaluate the expression for $\mathbf{X}$ in Eq. (9).

\section{Dependent versus redundant measurements}

It is important to understand the difference between dependent and redundant measurements.

\subsection{Dependent measurements}

Dependent measurements are measurements where the likelihood cannot be written as a product of independent likelihood functions, i.e.,

$f(\mathbf{d} \mid \mathbf{x}) \neq \prod_{i} f\left(\mathbf{d}_{i} \mid \mathbf{x}\right)$,

where the index $i$ may refer to measurements that could be of different kinds collected at different times or from different wells, and $\mathbf{x}$ denotes the static model parameters. The likelihood function is the probability of observing the data given the model parameters $\mathbf{x}$. The measurement error covariance matrix describes the dependency between measurements through correlations of the errors in the measurements. Such correlations arise in production rate data, e.g., when we interpolate measurements from a separator test in time through a rate-allocation procedure. Measurement errors are correlated in a large selection of data types, in particular when we use gridded, or processed data, but the correlations may also appear directly from the instrument used to collect the data.

\subsection{Redundant measurements}

By redundant measurements we mean measurements that contain some of the same information, e.g., two measurements of the temperature taken at approximately the same time and location will contain a significant amount of redundant information. The redundancy of two measurements is represented in the cross correlations in the covariance matrix for the predicted measurements $\mathbf{S S}^{\mathrm{T}}$, and redundancy of the measurements will imply poor conditioning of this matrix. In fact, if we collect two measurements at the same time and location, then the two corresponding rows in $\mathbf{H}$ will be identical, and $\mathbf{S S}^{\mathrm{T}}$ will be singular. Note that in observation design experiments it is common to collect the measurements such that the conditioning of $\mathbf{S S}^{\mathrm{T}}$ becomes optimal [3].

\subsection{Impact of dependency and redundancy of measurements}

To illustrate the impact of dependency and redundancy of measurements we consider the simple example of a scalar variable $\mathbf{x} \in \mathfrak{R}^{1}$ with variance $\mathbf{P} \in \mathfrak{R}^{1 \times 1}$ and we use the standard update equations from the Kalman filter written as

$$
\begin{aligned}
& \mathbf{x}^{\mathrm{a}}=\mathbf{x}^{\mathrm{f}}+\mathbf{P}^{\mathrm{f}} \mathbf{H}^{\mathrm{T}}\left(\mathbf{H} \mathbf{P}^{\mathrm{f}} \mathbf{H}^{\mathrm{T}}+\mathbf{R}\right)^{-1}\left(\mathbf{d}-\mathbf{H} \mathbf{x}^{\mathrm{f}}\right) \\
& \mathbf{P}^{\mathrm{a}}=\left(1-\mathbf{P}^{\mathrm{f}} \mathbf{H}^{\mathrm{T}}\left(\mathbf{H} \mathbf{P}^{\mathrm{f}} \mathbf{H}^{\mathrm{T}}+\mathbf{R}\right)^{-1} \mathbf{H}\right) \mathbf{P}^{\mathrm{f}} .
\end{aligned}
$$

We assume a prior value $\mathbf{x}^{\mathrm{f}}=0$ and prior variance $\mathbf{P}^{\mathrm{f}}=1$. 
In the case with one measurement $\mathbf{d}=1$ with variance $\mathbf{R}=1, \mathbf{H}=1$ is also a scalar, and from the update Eq. (13) we get

$\mathbf{x}^{\mathrm{a}}=\frac{1}{2}=0.5 \quad$ and $\quad \mathbf{P}^{\mathrm{a}}=\frac{1}{2}=0.5$.

Thus, we get the expected result centered between the first guess and the measurement values, and the error variance becomes half of the first guess and the measurement variance.

With two measurements of $\mathbf{x}, \mathbf{d}^{\mathrm{T}}=\left(\begin{array}{ll}1 & 1\end{array}\right)$ with uncorrelated measurement errors and variances equal to one, we define the measurement operator $\mathbf{H}^{\mathrm{T}}=\left(\begin{array}{ll}1 & 1\end{array}\right)$ and the inverse matrix in the update equation becomes

$\mathbf{H} \mathbf{P}^{\mathrm{f}} \mathbf{H}^{\mathrm{T}}+\mathbf{R}=\left(\begin{array}{ll}1 & 1 \\ 1 & 1\end{array}\right)+\left(\begin{array}{ll}1 & 0 \\ 0 & 1\end{array}\right)$.

It is clear that the redundancy of the information in the measurements leads to a singular $\mathbf{H} \mathbf{P}^{\mathrm{f}} \mathbf{H}^{\mathrm{T}}$, but the uncorrelated measurement errors make it possible to compute the inversion, and the result becomes

$\mathbf{x}^{\mathrm{a}}=\frac{2}{3} \approx 0.67 \quad$ and $\quad \mathbf{P}^{\mathrm{a}}=\frac{1}{3} \approx 0.33$.

We obtain the same result by assimilating the two data recursively, by starting from the result (14) and computing another update using the update equations from Eq. (13) with the second measurement. This example illustrates the well-known result that independent measurements, which have uncorrelated errors, can be processed recursively.

The redundancy may occur also using nearby-located measurements which leads to a poorly conditioned $\mathbf{H P H}^{\mathrm{T}}$ matrix resulting in unstable updates unless we use a pseudoinversion method when solving the update equations. Without truncation of the eigenvalue spectrum, we will divide by small numbers, and this leads to updates with large unphysical amplitudes. Thus, the redundancy is a problem related to the numerical computation of the update, but it will also allow us to subsample the time series of rate data when history matching reservoir models.

The situation changes when we condition on measurements with correlated errors. Assume that the two measurements of $\mathbf{x}, \mathbf{d}^{\mathrm{T}}=\left(\begin{array}{ll}1 & 1\end{array}\right)$, have an error correlation of 0.5 , i.e.,

$\mathbf{H P}^{\mathrm{f}} \mathbf{H}^{\mathrm{T}}+\mathbf{R}=\left(\begin{array}{ll}1 & 1 \\ 1 & 1\end{array}\right)+\left(\begin{array}{cc}1 & 1 / 2 \\ 1 / 2 & 1\end{array}\right)$.

The update then becomes

$\mathbf{x}^{\mathrm{a}}=\frac{4}{7} \approx 0.57 \quad$ and $\quad \mathbf{P}^{\mathrm{a}}=\frac{3}{7} \approx 0.43$

Thus, we notice how the measurements have a reduced impact because of the correlations in the errors.

This example illustrates two important points, first that the assimilation of redundant data leads to loss of rank in $\mathbf{S S}^{\mathrm{T}}$ and thus also $\mathbf{C}$, so care must be taken when computing the inverse of $\mathbf{C}$, second that neglecting correlated measurement errors leads to a too high impact of the measurements and possible ensemble collapse. Note that ensemble collapse may also occur in cases where there are more measurements than ensemble members $m>N$ since this may lead to a matrix $\mathbf{C} \in \Re^{m \times m}$ with rank less than or equal to $N-1$. In this case, it is necessary to use a pseudo-inversion algorithm when computing the inverse of $\mathbf{C}$. Also, conditioning on measurements with errors identically equal to zero will lead to loss of rank in the ensemble since all ensemble members interpolate these measurements.

\section{Rate data}

We consider production rate data given as produced oil, gas and water in $\mathrm{m}^{3} /$ day. The data are available at regular intervals, e.g., given as monthly or weekly averages. Also, there are additional rate data given every time there is an event such as a maintenance of a well or the introduction of a new well. Thus, there can be a large number of rate data for the multi-year history of a well. The rate data are used to force the simulation model when running in history mode, either by specifying one of the measured production rates of oil, gas or water, or perhaps most commonly, by specifying the produced reservoir-fluid-volume rate (RESV) corresponding to the observed flow rates of the different phases.

By forcing the model with the RESV rate, we give the model freedom to determine the relative volumes of produced oil, gas and water. The simulator will try to satisfy the specified RESV rates, but in some cases, the simulator is not able to produce the specified RESV rates at all times and wells, e.g., due to wrongly prescribed reservoir properties or an erroneous specification of the initial distribution of oil, gas, and water in the model. In the history matching, we condition the model on the observed rates of oil, gas, and water produced from each well. The first problem for the history matching is then to update model parameters so that the model produces the correct RESV rates for all wells. Secondly, by conditioning on the individual rate data, we attempt to update model parameters so that the model can produce the observed relative volumes of individual fluids for each well.

\subsection{Redundant information in rate data}

The information, available at different times, in a time series of rate data, is strongly correlated and redundant. We illustrate the redundancy of information in a time series of rate data in Fig. 1 where we have plotted the ensemble of predicted oil rates from the well D-3AH in the Norne model (to be discussed below). When looking at the ensemble 
Fig. 1 Ensemble correlations: The upper plot shows the ensemble predictions of oil rate for well D-3AH of the Norne model to be discussed below. The lower plot shows the correlation function of the ensemble of oil rates corresponding to day 1893
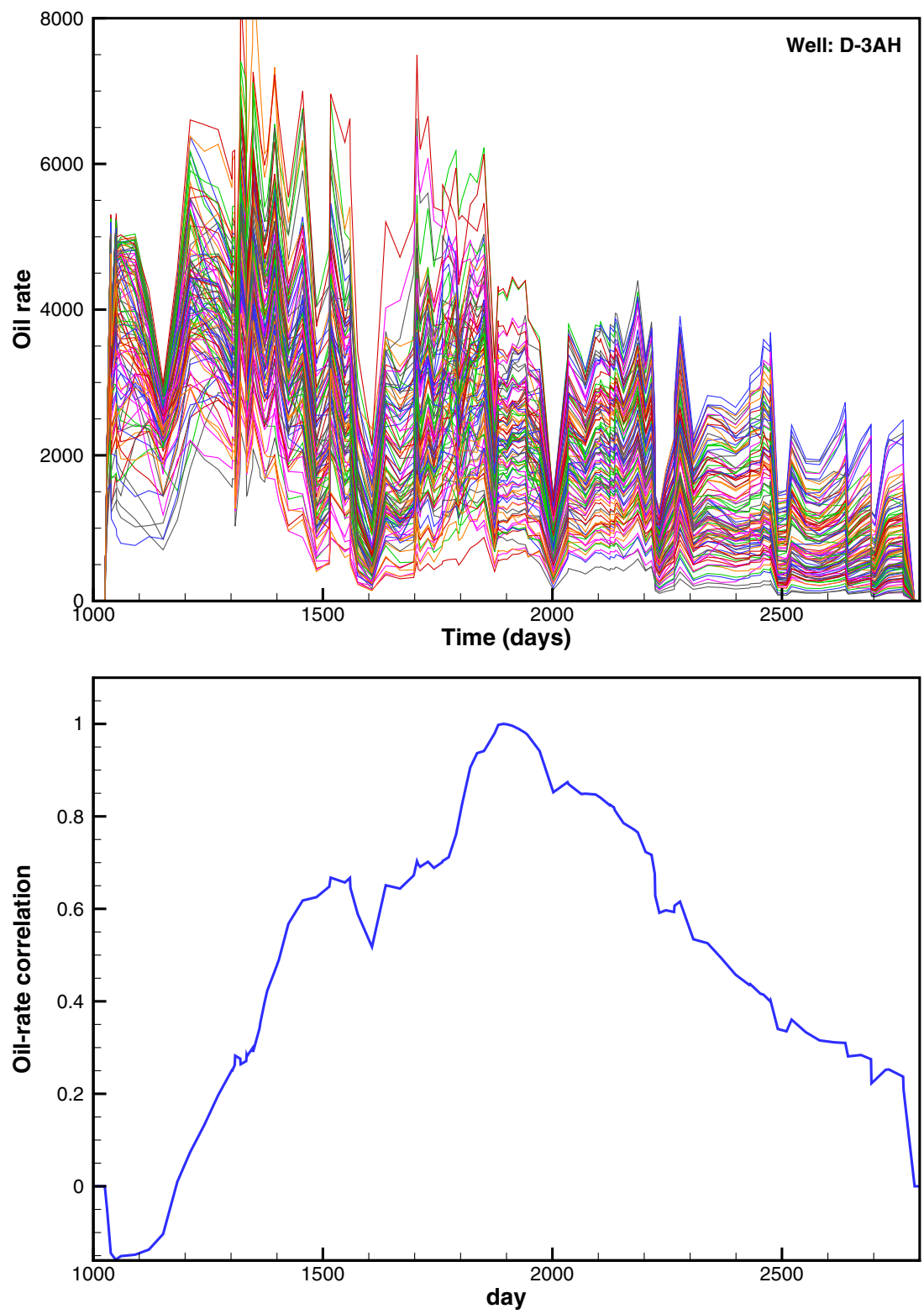

realizations, we can see that simulated oil rates from the ensemble members have somewhat similar behavior and this an indication of significant correlation in time. We show the correlation in time corresponding to day 1893 in the lower plot in Fig. 1. It is clear that there are correlations higher than 0.3 for at least two years before and after day 1893 . In fact, we observe similar correlations for all wells and all fluids in the Norne model.

The properties of reservoirs implicitly cause redundancy in the rate data. The reservoir is a static permeable rock, and a pressure difference between the reservoir and a production well causes fluids to flow towards the production well. The fluid flow is proportional to the pressure difference, meaning that the rock properties, e.g., between an injector and a producer, can be estimated at any time during the production phase, using both low and high pressuredifferences. It is the external forcing from the operation of wells that leads to the rapid changes we see in the rate data from real reservoirs, and dynamical processes cannot explain the variability in the rate data.

\subsection{Measurement error correlations in rate data}

In most variants of ES or EnKF, the measurement errorcovariance matrix $\mathbf{R}$ is specified to be diagonal [5, 17, 31], which states that measurement errors are uncorrelated. The main reason for assuming independent data and using a diagonal measurement error covariance matrix seems to be that most update algorithms require the inverse of $\mathbf{R}$, and this inversion becomes too expensive unless $\mathbf{R}$ is diagonal. 
Additionally, it is often not clear how to specify the offdiagonal terms representing correlations between different measurement errors. The use of a diagonal $\mathbf{R}$ will help stabilizing the inversion of $\mathbf{C}$ but for the wrong reason.

The assumption of uncorrelated measurement errors is not likely to be correct when conditioning on a time series of production rate data, mainly because the method used to generate the data is the same for the whole time series. Often, we base a time series of rate data on an extrapolation or interpolation in time, of allocated rates from regular but not very frequent separator tests. In the separator tests, one closes all wells except one and observe the production of oil, water, and gas, as a function of controls, e.g., gas-lift or valve openings. We can then plot the produced rates in an allocation table as a function of the controls. This procedure is repeated for all the wells one by one, and we thereby also ignore any interaction between the wells. We then extract the rates for each well from the allocation tables. Also, an allocation volume factor is used to scale up or down the total estimated production so that it matches the measured total production. In summary, an error in the allocation table will be the same for the whole time interval until the next separator test. Thus, if the algorithm produces too high rates at one time instant, it is likely that the rates are also too high at the next time instant and there is a presence of significant measurement error correlations.

The allocation procedures may also introduce error correlations between fluid rates from one well and error correlations between rates from different wells, but we have not considered such correlations in this paper.

In the EnKF the data are assimilated sequentially in time, and we implicitly neglect the error correlations in time. Thus, this approximation may explain the ensemble collapse and filter divergence observed in some studies using EnKF. Furthermore, when using smoothers, if we neglect the measurement error correlations, this will also lead to a collapse of the ensemble.

\subsection{Conditioning on accumulated production}

The production rates will contain errors from the allocation procedure used. The accumulated production is also determined from the same allocation procedure and will be prone to the same error sources. However, the error models for the accumulated production and the production rates will differ.

Suppose we have the observed production rates, $r_{i}$, for $i=1, \ldots, p$, where $p$ is the number of time intervals of length $\Delta t$. The accumulated production is then

$a_{i}=\Delta t \sum_{j=1}^{i} r_{j}$
Thus, the accumulated production contains redundant information in time since

$a_{i}=a_{i-1}+r_{i} \Delta t$.

However, the redundancy in the accumulated production will be handled by the update scheme as long as the numerical implementation is robust as discussed above.

For a case with four rates $r_{i}$ over constant time intervals $\Delta t$, we can write the accumulated rates as follows

$\left(\begin{array}{l}a_{1} \\ a_{2} \\ a_{3} \\ a_{4}\end{array}\right)=\Delta t\left(\begin{array}{llll}1 & 0 & 0 & 0 \\ 1 & 1 & 0 & 0 \\ 1 & 1 & 1 & 0 \\ 1 & 1 & 1 & 1\end{array}\right)\left(\begin{array}{l}r_{1} \\ r_{2} \\ r_{3} \\ r_{4}\end{array}\right)$.

Similarly, the inverse relation is

$\left(\begin{array}{l}r_{1} \\ r_{2} \\ r_{3} \\ r_{4}\end{array}\right)=\frac{1}{\Delta t}\left(\begin{array}{llll}1 & 0 & 0 & 0 \\ -1 & 1 & 0 & 0 \\ 0 & -1 & 1 & 0 \\ 0 & 0 & -1 & 1\end{array}\right)\left(\begin{array}{l}a_{1} \\ a_{2} \\ a_{3} \\ a_{4}\end{array}\right)$.

The matrix and its inverse are of full rank. Thus, there is no loss of information if we condition on the accumulated production rather than the rates. In fact, conditioning on the accumulated production is the same as conditioning on the rates since we use a full rank measurement matrix $\mathbf{H}$ as given by the matrix in Eq. (21), although additional correlations are introduced for the measurement errors for the accumulated production data, as shown below.

If the error covariance matrix for the rate data is diagonal meaning we have uncorrelated errors, and the variance is equal to $\sigma^{2}$, i.e., $\mathbf{R}=\sigma^{2} \mathbf{I}$, then the error covariance matrix for the accumulated production becomes

$\mathbf{H} \Delta t^{2} \sigma^{2} \mathbf{I H}^{\mathrm{T}}=\Delta t^{2} \sigma^{2}\left(\begin{array}{llll}1 & 1 & 1 & 1 \\ 1 & 2 & 2 & 2 \\ 1 & 2 & 3 & 3 \\ 1 & 2 & 3 & 4\end{array}\right)$.

Thus, we introduce correlations of the errors of accumulated production. If the errors in the rates are $100 \%$ correlated and $\mathbf{R}=\sigma^{2} 1$ where 1 is a matrix containing the number 1 for all elements, then we obtain the following error covariance matrix for the accumulated production data.

$\mathbf{H} \Delta t^{2} \sigma^{2} 1 \mathbf{H}^{\mathrm{T}}=\Delta t^{2} \sigma^{2}\left(\begin{array}{llll}1 & 2 & 3 & 4 \\ 2 & 4 & 6 & 8 \\ 3 & 6 & 9 & 12 \\ 4 & 8 & 12 & 16\end{array}\right)$.

The interesting point here is that for rate errors uncorrelated in time, the standard deviation of the error in $a_{i}$ will equal $\sqrt{i}$, and given a constant value of $r_{i}=r$ for all $i$ we observe that the relative error in $a_{i}$ (i.e., the standard deviation of $a_{i}$ divided by $a_{i}$ ) becomes $(r \sqrt{i})^{-1}$, that is, it approaches zero proportional to $1 / \sqrt{i}$. In the extreme case with $100 \%$ correlation, the relative error in $a_{i}$ will be proportional to 1 . 
Thus, the use of accumulated production leads to a lower relative variance of the errors than what we will have when we condition on rates. If one wants to avoid introducing the time correlation in the accumulated data, one can use the accumulated production over separate time periods.

\subsection{Consistent conditioning on rate data}

We have seen in Section 4.1 that there may be a significant amount of redundant information in rate data that leads to loss of rank of $\mathbf{C}$. Furthermore, due to the allocation methods used to obtain the rates, it is likely that there are error correlations in time for the measurements that must be modeled and represented in $\mathbf{R}$. Finally, the rate data are implicitly used to force the reservoir model through the derived RESV data that control the time variability of the production. Thus, the conditioning of reservoir models on rate data is non-trivial and must be done with care to ensure consistency.

The typical approach, when conditioning reservoir models on rate data using ensemble smoothers and filters, is to select weekly, bi-weekly, or monthly rate data, together with a diagonal measurement error covariance matrix. Thus, we ignore possible error correlations in the data. For this approach to be consistent, it is necessary to model the measurement error correlations to build $\mathbf{R}$ and also use stable numerical schemes in the inversion [12]. However, the error statistics of the rate data are poorly known, and it is challenging to specify the error covariances correctly. An alternative is to subsample the rate data to an extent where error correlations in the data are negligible, and we can use a diagonal $\mathbf{R}$. The subsampling may also lead to a loss of information in the conditioning. We should sub-sample the rate data such that we do not include data containing significant redundant information, and we define significant as being correlated above a certain threshold, e.g., 0.2-0.3. This approach also leads to better conditioning of $\mathbf{C}$ and most standard implementations of the update scheme should work well.

The approach taken in this paper uses subsampling of the data, and we exemplify it by conditioning the model only on the total accumulated production of oil, gas, and water, for each well, and specified at the final time of the history. The total accumulated production contains information about how much oil, water, and gas the reservoir contains and how the oil, water, and gas are flowing. The reservoir consists of static properties, and the flow properties will typically not change with time, although the introduction of new wells will change the flow and provide additional information. Thus, we control the time variability of the production by specifying the RESV rates in the simulator.
Furthermore, we control the relative production from each well and also the total produced volumes of oil, water, and gas by conditioning on the total accumulated production of oil, water, and gas.

With the proposed approach we reduce the number of measurements to three per well, so the total number of measurements will in many cases be of the same order as the number of ensemble realizations. Furthermore, we may not have to deal with correlated measurement errors between the total accumulated production from different wells. Also, the numerical computation of the update should be trivial, and we do not have to worry about the same data being used both in the conditioning and the forcing of the model.

\subsection{Model errors}

An important issue for future studies concerns the representation of stochastic noise in the rate data used to force the ensemble realizations. The rates are obtained from observations and contain errors, but these errors are traditionally not taken into account when running reservoir simulators. The inclusion of stochastic white or red noise in the rate data leads to an increased predicted ensemble variance which would give rise to a stronger impact of the measurements in the update step. However, the current formulation of the history-matching problem solved by iterative ensemble smoothers cannot account for errors in rate data, or any form of model errors, since the methods are developed based on an assumption of zero model errors.

Table 1 Prior distributions used for the uncertain parameters

\begin{tabular}{lll}
\hline Parameter & Dimension & Distribution \\
\hline PORO & 44927 & NOR \\
PERMX & 44927 & LOG-NOR \\
MULTFLT & 53 & LOG-UNI [0.00001: 1.0] \\
MULTRGT & 3 & LOG-UNI [0.00001 : 1.0] \\
MULTZ & 6 & LOG-UNI [0.00001 : 1.0] \\
RELPERM KRW & 4 & UNI [0.8 : 1.5$]$ \\
RELPERM KRG & 4 & UNI [0.8 : 1.0$]$ \\
OWC:Garn (C,D) & 1 & NOR N $(2682.0 \mathrm{~m}, 5 \mathrm{~m})$ \\
OWC:Garn (G) & 1 & NOR N $(2585.5 \mathrm{~m}, 5 \mathrm{~m})$ \\
OWC:Garn (E) & 1 & NOR N $(2638.0 \mathrm{~m}, 5 \mathrm{~m})$ \\
OWC:Ile, Tilje (G) & 1 & NOR N(2400.0 m, $5 \mathrm{~m})$ \\
OWC:Ile, Tilje (C,D,E) & 1 & NOR N (2693.3 m, 5 m) \\
\hline
\end{tabular}

For porosity and permeability we have given the statistics in Table 2 . KRW and KRG are the endpoint scaling parameters for the water and gas relative permeability curves. Oil-water contacts for the four segments C, D, E, and G in the three formations Garn, Ile, and Tofte are also included 
Table 2 The table shows the vertical correlation and averages used to construct porosity and permeability fields

\begin{tabular}{llll}
\hline Layer & Vertical & Average & Average \\
\hline & corr & Poro & $\log ($ Permx $)$ \\
1 & 0.00 & 0.27 & 6.09 \\
2 & 0.00 & 0.25 & 5.48 \\
3 & 0.00 & 0.21 & 3.65 \\
5 & 0.00 & 0.23 & 4.54 \\
6 & 1.00 & 0.23 & 4.54 \\
7 & 0.77 & 0.24 & 4.72 \\
8 & 1.00 & 0.24 & 4.72 \\
9 & 0.00 & 0.25 & 6.02 \\
10 & 0.00 & 0.26 & 6.43 \\
11 & 0.00 & 0.24 & 5.22 \\
12 & 0.00 & 0.23 & 4.31 \\
13 & 0.90 & 0.29 & 6.71 \\
14 & 0.84 & 0.27 & 5.62 \\
15 & 0.88 & 0.24 & 4.65 \\
16 & 0.00 & 0.23 & 5.98 \\
17 & 0.00 & 0.20 & 4.34 \\
18 & 0.00 & 0.25 & 6.46 \\
19 & 0.00 & 0.20 & 4.70 \\
20 & 0.00 & 0.24 & 5.82 \\
21 & 0.00 & 0.19 & 4.83 \\
22 & 0.00 & 0.26 & 6.85 \\
\hline & & &
\end{tabular}

In column 2 the strength of the vertical correlation denotes how fields in a particular layer are correlated to the fields in the layer above. Both the average values for porosity and permeability and the vertical correlations were calculated from the model released by Statoil. The correlations were calculated from porosity and permeability together, and we only kept the correlations above 0.70

\section{Application with the Norne full-field model}

The reservoir model used in this study is the full-field Norne model, which has been made available by Statoil through the IO Center at the Norwegian University of Science and Technology (NTNU). The model is a version of the real field model that is operated by Statoil and with ENI and Petoro as partners. [6] discuss the model details in a previous application of an iterated ensemble smoother.
The experiment presented in the current paper, repeats the experiment in [6], but with some differences.

For the parametrization, we have included the porosity and permeability of all active grid cells, fault multipliers, region multipliers, vertical transmissibility between six layers, the relative permeability, and contact depths, as parameters to history match (see Table 1). Contrary to the study by [6] we have not included net-to-gross parameters since there is not a clear distinction between updating porosity and net-to-gross. The porosity and permeability fields are simulated based on average values, uncertainties, and Gaussian variograms with horizontal and vertical decorrelation lengths extracted from the geological interpretation of the reservoir. See Tables 2 and 3 for a list of parameters. The vertical permeability is given as a multiplier times the horizontal permeability. Another difference from [6] is that we assumed the vertical transmissibilities to be constant in each layer since it is then easier to ensure that there is either communication or no communication between the layers. With horizontally varying transmissibility, there is a risk for communication between layers at random locations while in reality there is a sealing boundary between the layers. We also used loguniform distributions for all the multipliers while [6] used log-normal priors. The reason for this change is to give a higher probability for closed faults and to avoid getting multipliers larger than one.

While [6] conditioned on bi-monthly rates of produced oil, water, and gas, for 22 wells, the main difference in our study is that we have only included the accumulated total production of oil, water, and gas taken at the final time in the conditioning. Thus, while [6] had three rate measurements for each of 22 wells at up to 62 instants in time, in total about 2000 observations, we have used three measurements from each of 22 wells and are only conditioning on a total of 66 observations. We could also have subsampled the rate data directly at appropriate time intervals. However, we do not trust the rate data enough to use a small set of subsampled data values. There are significant errors in the rate data due to the allocation procedure used, and we believe that the errors in total production are significantly lower since these data are measured. Thus, we exploit the fact that the external forcing from the operation of existing wells and the introduction of new wells causes the variability we see

Table 3 The table shows correlation between porosity and permeability, standard deviations, maximum and minimum values for porosity and permeability, and the decorrelation length used when constructing the porosity and permeability fields

\begin{tabular}{llllllll}
\hline $\begin{array}{l}\text { Correlation } \\
\text { Poro, } \log (\text { Permx })\end{array}$ & $\begin{array}{l}\text { Std. Dev. } \\
\text { Poro }\end{array}$ & $\begin{array}{l}\text { Std. Dev. } \\
\log (\text { Permx })\end{array}$ & $\begin{array}{l}\text { Min } \\
\text { Poro }\end{array}$ & $\begin{array}{l}\text { Max } \\
\text { Poro }\end{array}$ & $\begin{array}{l}\text { Min } \\
\text { Permx }\end{array}$ & $\begin{array}{l}\text { Max } \\
\text { Permx }\end{array}$ & $\begin{array}{l}\text { Decorrelation } \\
\text { length (m) }\end{array}$ \\
\hline 0.70 & 0.05 & 1.00 & 0.01 & 0.4 & 0.0 & 10000 & 2500.0 \\
\hline
\end{tabular}


in the rate data. Also, we want to test the hypothesis that with the reservoir model being a static medium, then the conditioning on the total accumulated production of fluids from each well, combined with the forcing of the model by RESV that introduces the time variability in the production, is sufficient to history match the model. Finally, we want to avoid conditioning on the same rate data that are used to force the model. We have not conditioned on RFT data as was done in [6].
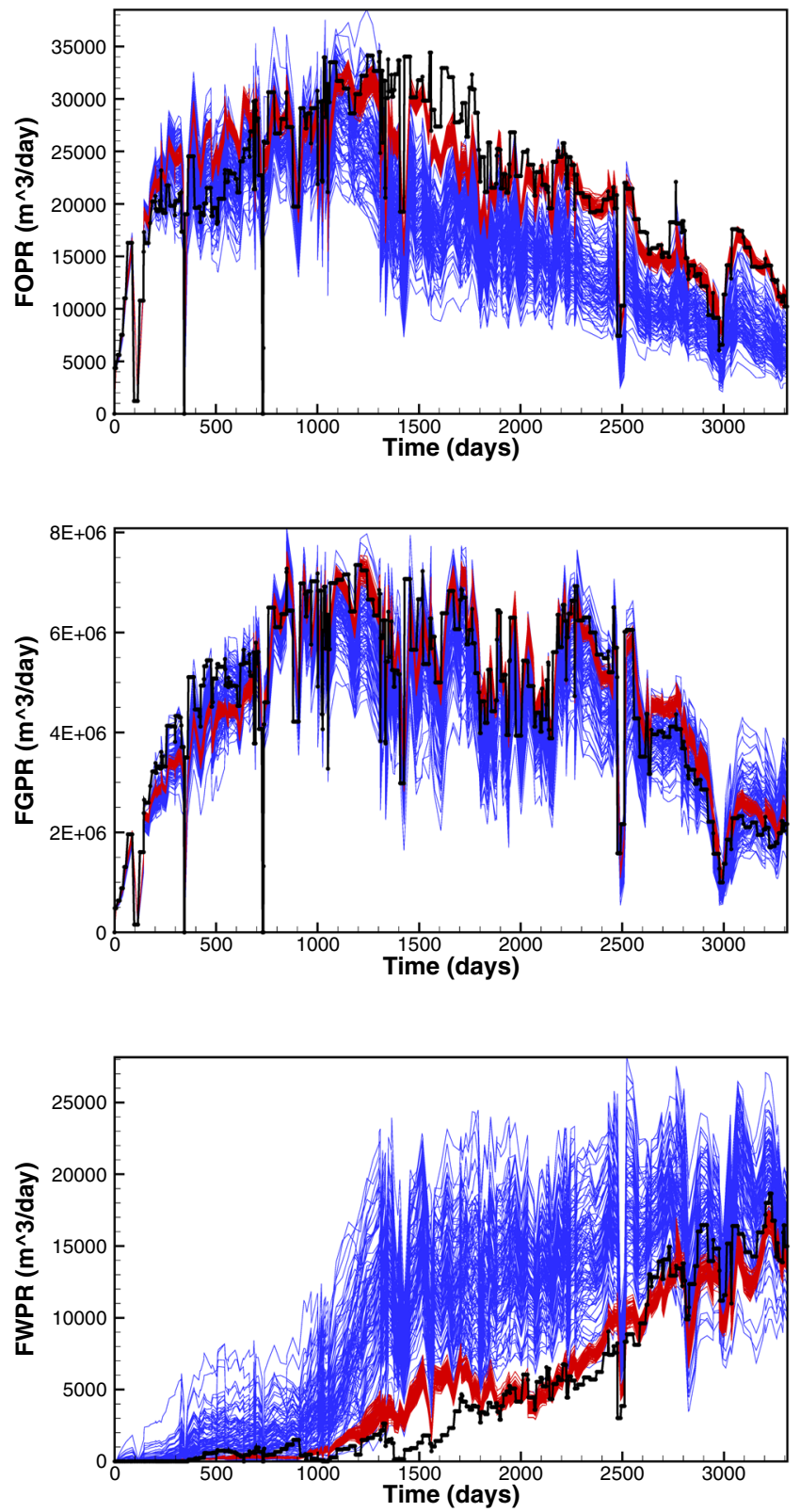

Fig. 2 ESMDA 16: Field totals. The figure shows the field production using ESMDA with 16 steps. The black line is historical data, the blue lines show the simulated production from the initial ensemble, and the red lines show the simulated production from the updated
Another significant difference is that we did not use any localization and computed a global update while [6] included localization to prevent ensemble collapse and to reduce the effect of spurious correlations. Since localization implies that we search for the solution in a larger space, there is a possibility that we restrict our update too much, by only searching for solutions contained in the space spanned by the original ensemble, when we decide not to localize the update. After all, we are solving for approximately
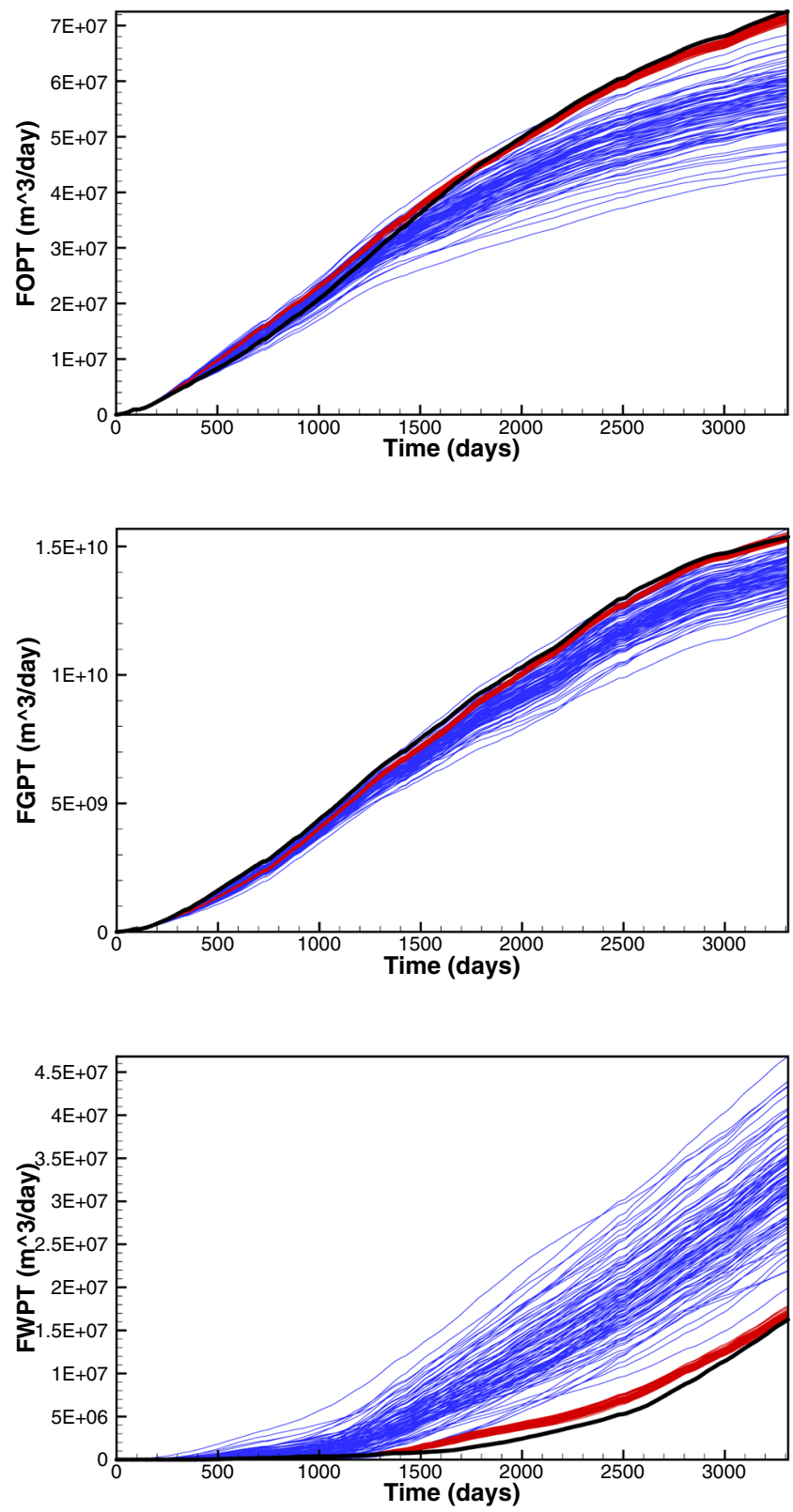

ensemble. The left column contains the rates of oil (top), gas (middle) and water (bottom), and the right column shows the corresponding total production 
90000 unknowns in a space spanned by only 100 ensemble members. However, we want to show that we can compute a global update without ensemble collapse and that the total accumulated production represents the information in the data.

The assimilation software used in this study is the Ensemble Reservoir Tool (ERT), developed by Statoil, which is an open-source software package available from Github. ERT is a powerful software where the main features include the administration of multiple model simulations and the provision of state-of-the-art implementations of popular ensemble smoothers, e.g., ES, ESMDA, and IES.
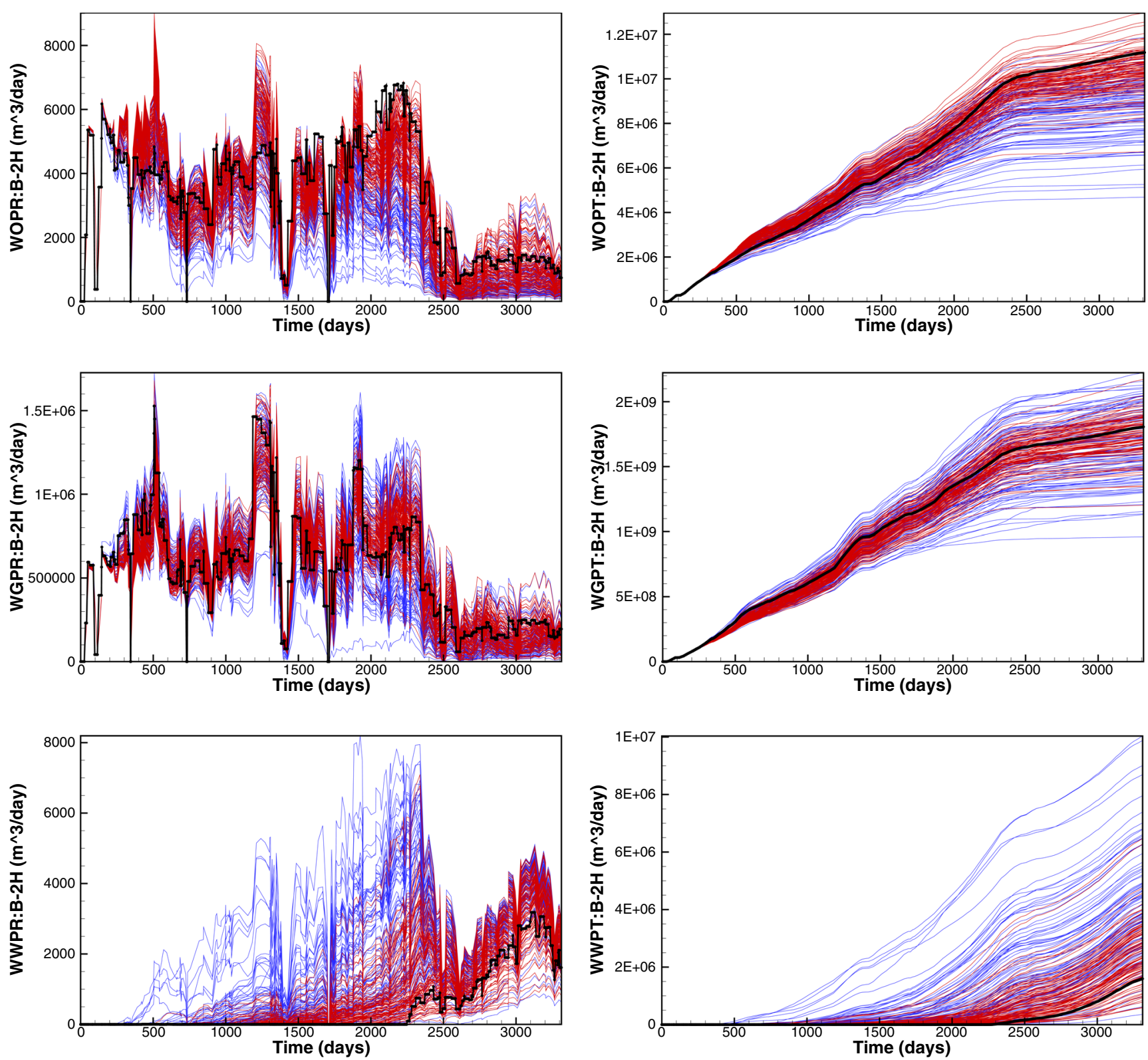

Fig. 3 ES: B-2H. The figure shows the results for well B-2H using ES. See Fig. 2 for description 
As mentioned above we assimilated three observations from each of 22 wells, and thus have a total of 66 measurements. The measurement error used was five $\%$ of the measurement value with a minimum of $100 \mathrm{~m}^{3}$. With 100 model realizations in the ensemble and mostly independent information from measurements without correlated errors, we do not expect any numerical difficulties from the actual inversion in these experiments.

When examining the prior ensemble for all wells, we observe that there are some wells where the model with its parametrization is not able to reproduce the observed rates (see, e.g., the water rate in Fig. 8 discussed below.) We did not attempt to significantly change or improve the model parametrization to better simulate these wells, since the scope of this work is not to obtain the best possible Norne model, but rather to test a methodology. It has also become clear from interaction with Statoil that significant structural changes are needed for the model to simulate all wells correctly. We used the same initial ensemble for the four experiments.

In general, we observe a substantial improvement in the data match for all experiments after history matching. We show the total field production for ESMDA 16 in Fig. 2 and all cases lead to history-matched models that matched the field totals, although ES gives a too large ensemble spread as will be illustrated next.

It is clear that ES produces results with a too large variance, as seen for the well $\mathrm{B}-2 \mathrm{H}$ in Fig. 3. For this
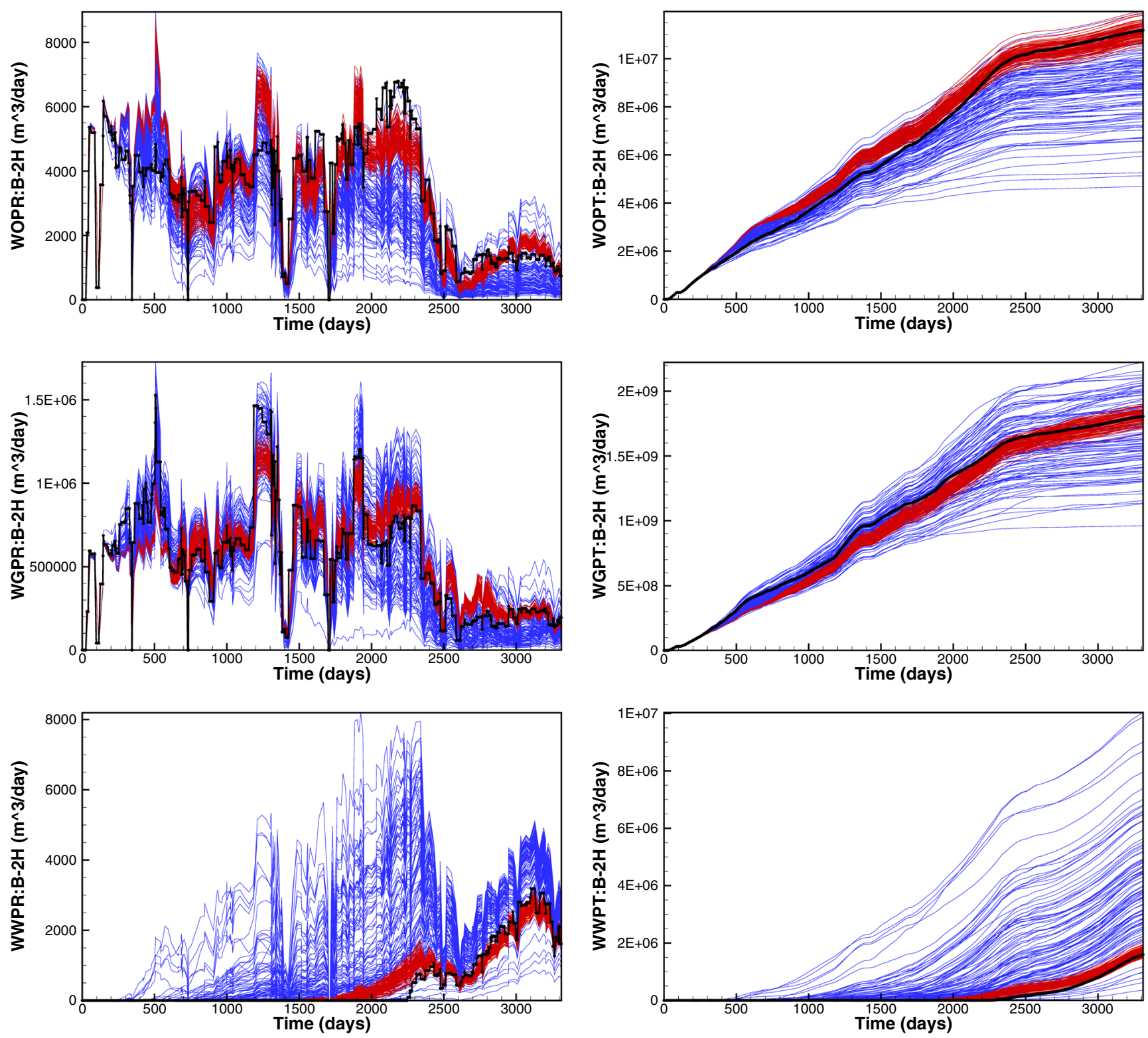

Fig. 4 ESMDA 8: B-2H. The figure shows the results for well B-2H using ESMDA with 8 steps. See Fig. 2 for description 

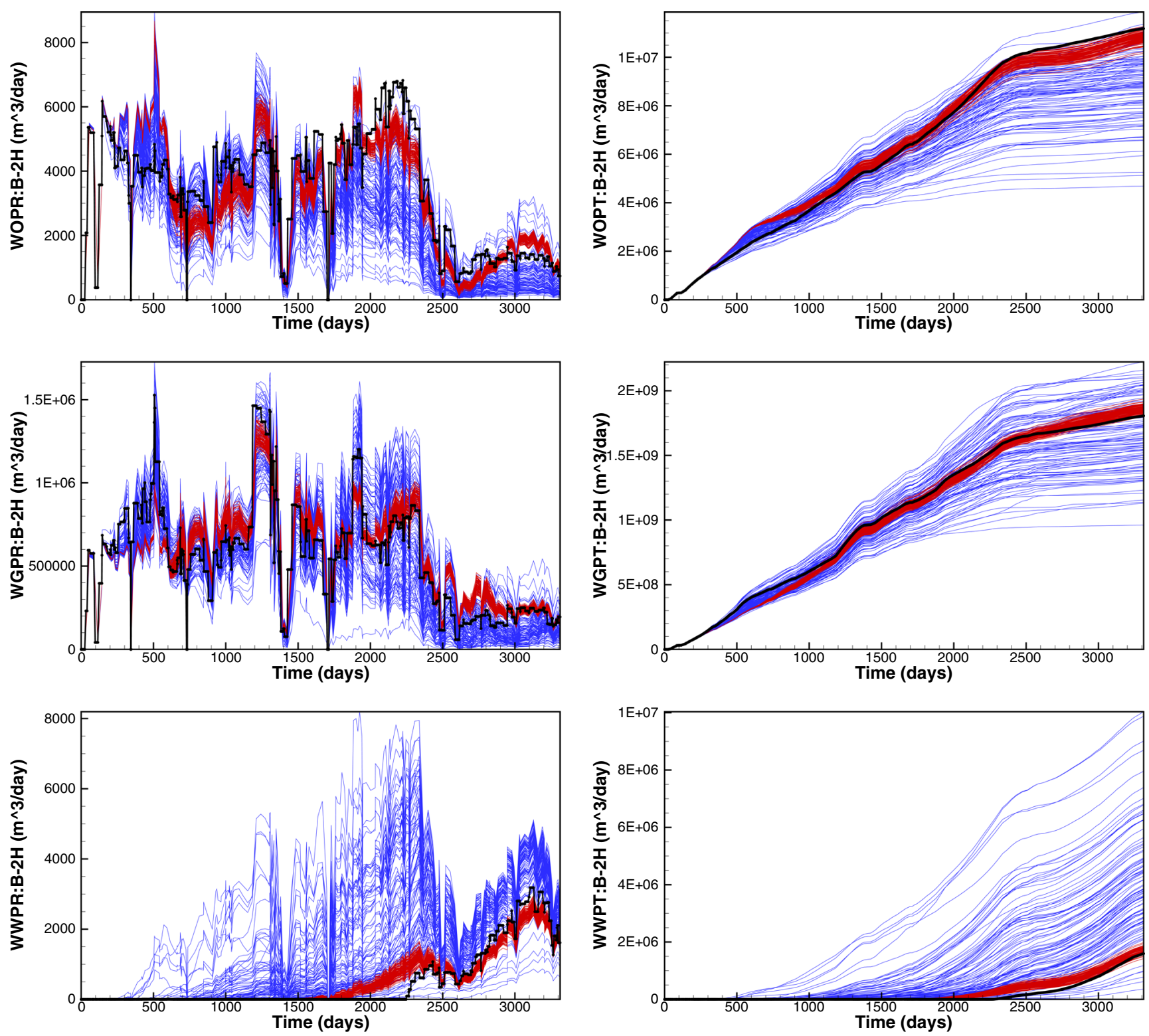

Fig. 5 ESMDA 16: B-2H. The figure shows the results for well B-2H using ESMDA with 16 steps. See Fig. 2 for description

well, ESMDA 8, ESMDA 16 and IES 15 obtained a reasonably good match to the historical data as shown in Figs. 4, 5 and 6. For the iterative methods, the rates are close to the historical data for most of the time. We see some discrepancies although the total production fits well with the observations. From the experiments, it is clear that we retain a non-zero variance in the ensemble both using ESMDA and IES, and we did not observe any tendencies to ensemble collapse. We are considering a nonlinear problem, and we use approximate methods for sampling the posterior conditioned distribution as given by Bayes' theorem. Thus, we can only claim that the results are consistent with the data and assumed uncertainties for the uncertain parameters within the approximations we make in the different ensemble smoothers. Thus, it makes sense also to use more than one method to be able to verify the results. In a real history-matching case the actual values of the parameters are unknown, and we know there are errors in the rate data. Thus, to examine the quality of the results, we are concerned whether the conditioning on the total accumulated production leads to a reasonable match to the time series of rate data and whether the ensemble spread is large enough to contain most of the rate data.

From a closer examination of the results in Fig. 3, we see that for most of the time the rate data are contained within the spread of the ensemble. However, we also notice that we have a slightly too high production of oil around day 500 , and day 1250 , and a correspondingly too 

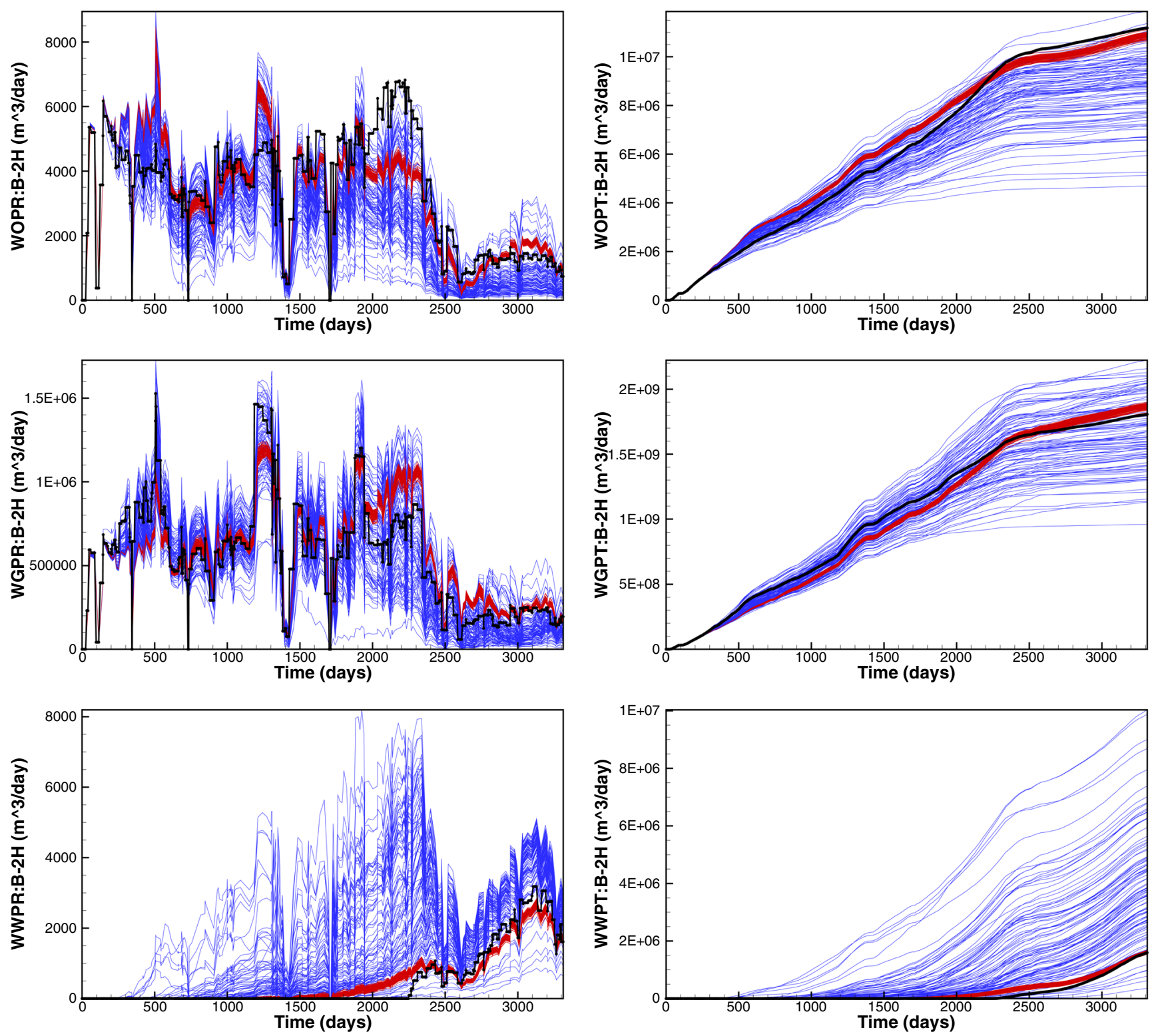

Fig. 6 IES 15: B-2H. The figure shows the results for well B-2H using IES with 15 iterations. See Fig. 2 for description

low production around day 2200 . These deviations match a lower and higher production of gas to maintain the imposed RESV rate. When conditioning only on the final total production, the measurements will not provide any information that can change this behavior. Thus, maybe a better match could be achieved by including a few additional OPT observations for this well. However, it is not clear that the addition of data points will lead to a better total match for all wells. We expect that to improve the match to the rate data at the few locations where the ensemble deviate from the observations we instead need to change the parametrization, i.e., add new uncertain parameters that will allow the model to match the observations we miss. In fact, we notice that our prior ensemble has some deficiencies that we should fix before we start the conditioning. The total production in Fig. 2, indicates that we produce too little oil and gas, and too much water. Thus, it might be useful to further calibrate some global parameters such as mean permeability and oil-water contacts to obtain a better prior ensemble. The next step concerns calibration of individual wells where the prior ensemble is unable to represent the observations. It is highly recommended to invest a significant part of the work in this pre-calibration phase since this will simplify the final assisted history matching.

It is interesting to see that all three iterative experiments produce results that are similar in features and behavior. If there is a difference between the history-matched results and 
the measured, the difference usually appears in both IES and ESMDA. Thus, we attribute the differences in outcomes to the parametrization rather than the history-matching method used.

The main difference observed between the different methods is that ESMDA with eight steps produces a lower variance than ES, ESMDA 16 has lower variance than ESMDA 8, and finally, IES has even lower variance than ESMDA 16. Thus, the multiple steps used in MDA are essential to reduce the variance, and the variance reduction increases with the number of steps. However, in ESMDA it is not clear how many steps we need for convergence, or what a converged result will be.

IES converged in 15 iterations, and the algorithm stopped when reaching a convergence criterion where the relative change in the misfit to measurements, between iterations, is smaller than a specified small value. We used the criterion $\left(S_{l}-S_{l-1}\right) / S_{l-1}<0.0001$ with $S_{l}=\operatorname{trace}\left(\left(\mathbf{d}_{l}-\right.\right.$ d) ${ }^{\mathrm{T}} \mathbf{R}^{-1}\left(\mathbf{d}_{l}-\mathbf{d}\right)$ ) for iteration $l$, where $\mathbf{d}_{l}$ represents the predicted measurements, $\mathbf{d}$ is the observations, and $\mathbf{R}$ is the measurement error covariance matrix [5].

ESMDA is not an iterative scheme as IES. In ESMDA one split the ES update into a predefined number of steps to reduce the errors introduced by nonlinearity and associated linearizations used in the method. We do not know how many steps we need to obtain convergence of ESMDA, but most applications use eight to sixteen steps. However, see [14] for some simple convergence test on ESMDA. Thus we attribute the larger variance in ESMDA
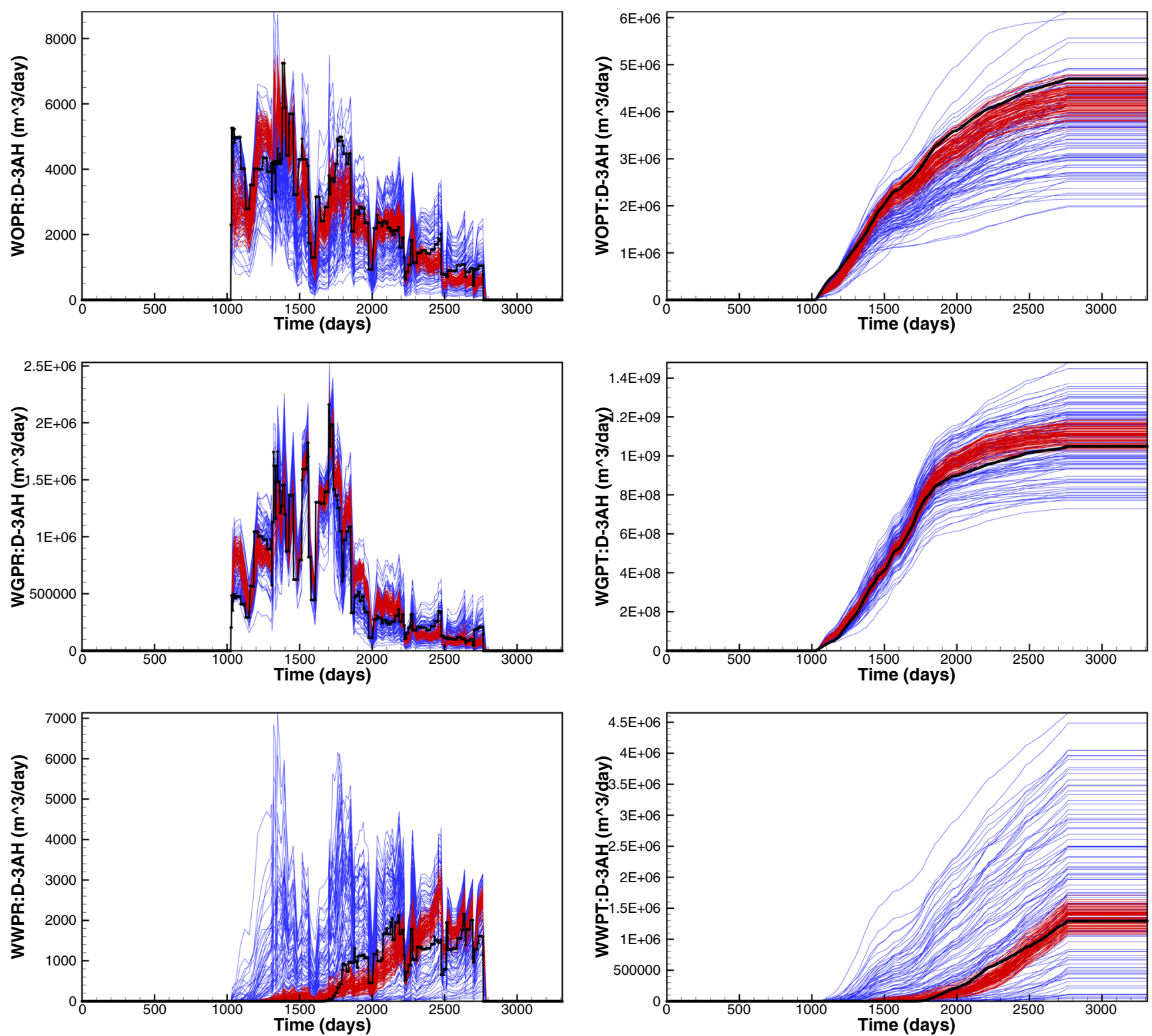

Fig. 7 ESMDA 8: D-3AH. The figure shows the results for well D-3AH using ESMDA with eight steps. See Fig. 2 for description 

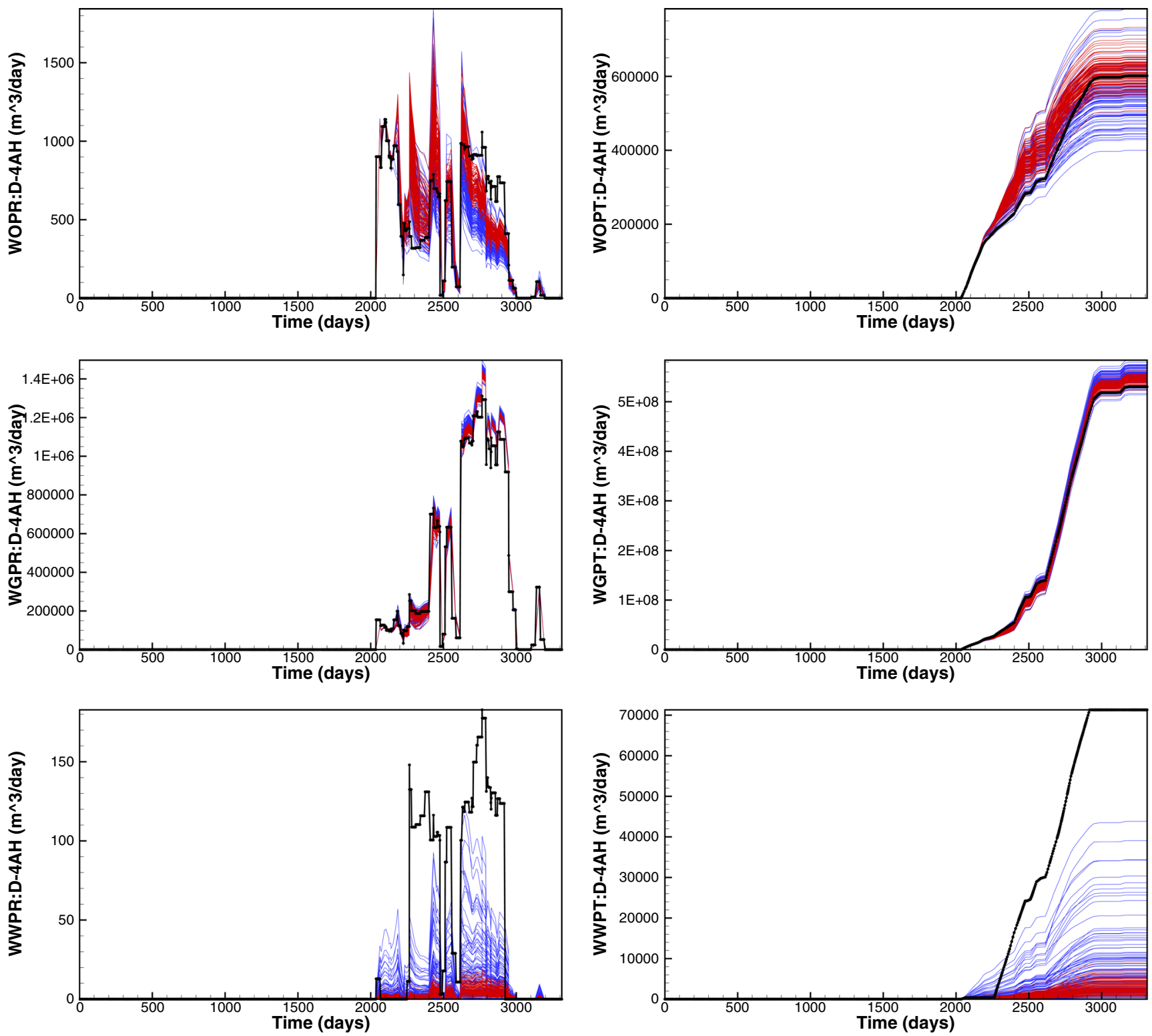

Fig. 8 ESMDA 8: D-4AH. The figure shows the results for well D-4AH using ESMDA with 8 steps. See Fig. 2 for description

compared to IES to the properties of the two different methods and partially to the number of ESMDA steps used.

While IES minimizes an ensemble of cost functions directly by a gradient method, ESMDA approximate the solution by a recursion of the ES equation, and for nonlinear problems, these solutions will differ.

From the figures, we also notice that the plotting of accumulated production makes it easier to analyze the results since any excess or too low production is immediately visible. When examining only the rates, it is hard to say if there is a good match or not due to the erratic behavior of the time series of rate data. In Fig. 7 we show another excellent result for ESMDA 8, and this well better illustrates that we maintain a finite realistic variance in the posterior ensemble and avoid ensemble collapse.

An interesting feature observed from the experiments is related to a pre-screening of observations in ERT. We consider observations that are located far from the prior

Table 4 Wells for which the measurement of WWPT were excluded in ERT

\begin{tabular}{llll}
\hline & IES 15 & ESMDA 8 & ESMDA 16 \\
\hline B-4H & $\mathrm{x}$ & & \\
D-4AH & $\mathrm{x}$ & $\mathrm{x}$ & $\mathrm{x}$ \\
E-3H & $\mathrm{x}$ & $\mathrm{x}$ & $\mathrm{x}$ \\
K-3H & & $\mathrm{x}$ & $\mathrm{x}$ \\
\hline
\end{tabular}


ensemble compared to the magnitude of the standard deviations in the ensemble and the measurements as outliers, and we exclude them from the conditioning. This feature is implemented to avoid conditioning on a measurement that the ensemble and current parametrization cannot represent and which may lead to unphysical updates of the ensemble. We define the innovation as the distance between a measurement and the mean of the ensemble-predicted measurement. The criterion used, excludes a measurement if the innovation exceeds $3\left(\sigma_{\text {obs }}+\right.$ $\left.\sigma_{\text {ens }}\right)$ where $\sigma_{\text {obs }}$ and $\sigma_{\text {ens }}$ are respectively the standard deviations of the measurement and the ensemble-predicted measurement. With the settings used in the experiments, a few observations were not conditioned on, see Table 4.
The ERT software excluded the water measurement for well $\mathrm{D}-4 \mathrm{AH}$ in all three experiments, and the simulated water production does not match the historical data, see Fig. 8. From the figure, it is clear that our prior ensemble is not able to reproduce the water rates for this well, and since the ERT excludes the total accumulated water datum from the conditioning, it is not possible to match the water for this well. The recommendation is to go back and improve the model so that the prior ensemble better represents the observed water production for this well. Thus, this feature of ERT leads to some wells not being correctly matched, but maybe relaxing the conditioning of some wells leads to a better match for other wells due to the use of a too simplified parametrization.
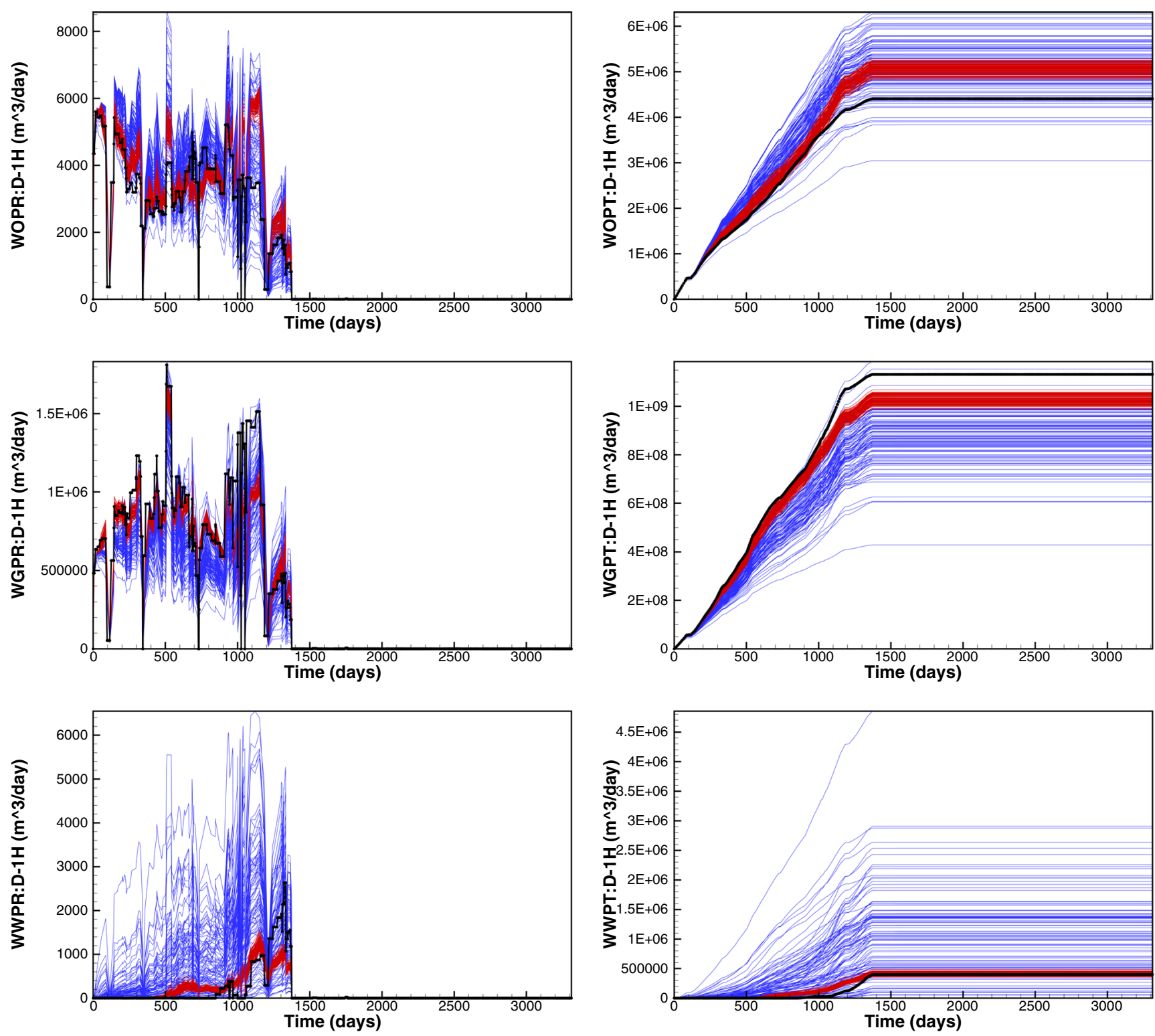

Fig. 9 ESMDA 16: D-1H. The figure shows the results for well D-1H using ESMDA with 16 steps. See Fig. 2 for description 

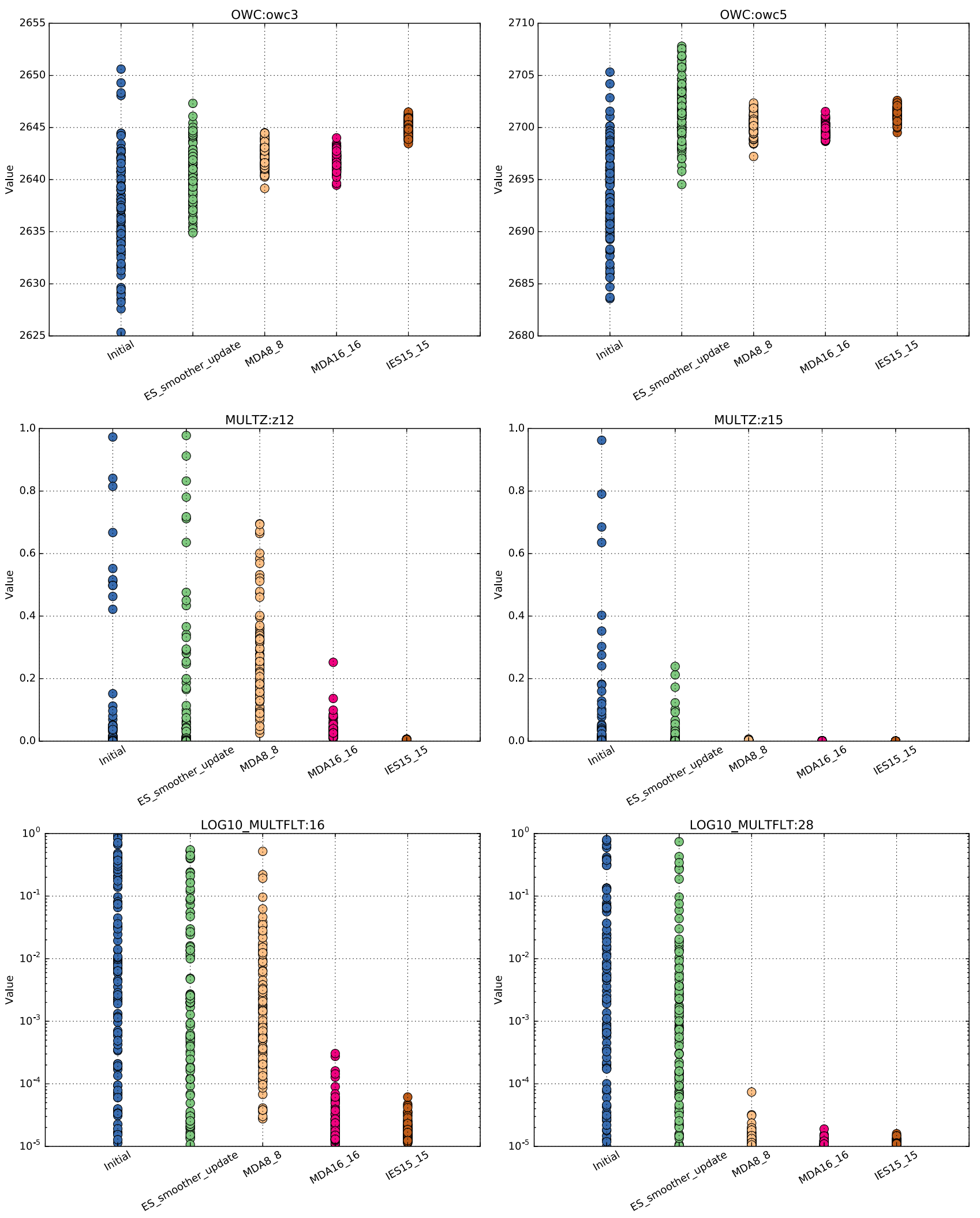

Fig. 10 The figure shows some selected parameters for the initial ensemble and the updated ensembles using ES, ESMDA 8, ESMDA 16 and IES. The upper plots show oil-water contacts in the E-segment of the Garn formation (left) and the C-, D-, and E-segments of the Ile

to Tilje formations. The middle plots show vertical multipliers in layer 12 (left) and 15 (right), and the lower plots show the logarithm of the multipliers of the faults C-08 (left) and C-20 (right) 
For some of the wells, the total simulated production did not match the measurements, see Fig. 9, although for most of the time the rates are close to the historical production. This result is an indication that we use a too limited parametrization.

We plot some of the updated parameters in Fig. 10 where we include results from ES, ESMDA 8, ESMDA 16 and IES. The figure shows two oil-water contacts, two vertical multipliers, and two fault multipliers. ES gives the weakest update to the parameters, which explains the more substantial variance in the ES predicted posterior ensemble. IES provides the strongest updates, but probably ESMDA will also result in a lower variance if we use the method with more update steps. For some of the parameters ESMDA 8, ESMDA 16 and IES give very similar results, and for other parameters, the spread in ESMDA 8, and sometimes ESMDA 16, is more extensive than for IES. This result is an indication that we need to use a larger number of steps for convergence of ESMDA. There were also a few parameters where the different methods converged to completely different results, like open versus closed faults, and that is an indication of the bimodality of the problem where a different combination of parameters results in a similar match to the data.

In an entirely controlled synthetic experiment, it makes sense to compute statistics on the results, since then the synthetic data are consistent with the model and uncertainty parametrization used. One can then compare different methods and their properties using statistical measures. However, in the current case, we already know that the parametrization is insufficient and there are likely to be additional sources of errors that are not accounted for (e.g., we ignore all uncertainties in the structural model). The standard measures such as innovation statistics can be computed, but they would only show us that we are inconsistent with the prior uncertainties. Additionally, we have also neglected the model errors. We already see that we are unable to match some wells, due to an insufficient parametrization, while for other wells we obtain what we would characterize as a reasonable match. We think that for real cases we are still more concerned about getting a result that is better than the prior when used in the reservoir management process and we believe that we have achieved this.

\section{Summary}

In this paper, we have pointed out that rate data include a dependency in time, i.e., the measurements have errors correlated in time. This correlation is mainly a result of the rate-allocation procedures used to generate the data. Also, we have illustrated how a time series of rate data contains redundant information. The data dependency and redundancy in time lead to particular problems when we use ensemble smoothers to condition the model on the data. First of all, we cannot neglect the off-diagonal elements in the measurement error covariance matrix, and we must correctly model them. Furthermore, we need to use inversion algorithms that can handle a nondiagonal measurement error covariance matrix. Secondly, the redundancy of information in the data leads to poor conditioning of the inversion and special care must be taken when choosing an inversion algorithm. For the full inversion problem with large data sets, the ensemble subspace inversion algorithm from [12] handles both the rank problem and the use of off-diagonal elements in the measurement error covariance matrix.

On the other hand, the redundancy of information in a time series of rate data allows us to use a subsample of rate data in the conditioning. We examined ES, ESMDA, and IES in an example with the Norne full-field model, where we only conditioned the model on the final total accumulated production of oil, gas, and water for 22 wells, and we used only 66 measurements for the history matching. We obtained an acceptable match for most wells, using both ESMDA and IES, which proves that the total accumulated production represents a significant part of the information in the rate data.

In our experiments, the conditioning on the total accumulated production of each phase updates the model parameters so that the model produces the correct volumes of different phases for each well, while the forcing of the model with RESV rates introduces the correct time variability in the rates for each well. We have considered an extreme example of subsampling when using only the final accumulated production of each phase and maybe using accumulated production over shorter time intervals could have further improved the match, although there seems to be a higher potential for improvement by working with the prior parametrization.

We have shown that reservoir models, due to their physical properties, may be history matched using only the total accumulated production for each well. In fact, considering the difficulties in prescribing and inverting a correct measurement error covariance matrix, as well as numerically solving the low-rank inversion problem in the update, it is probably wise to start with a more straightforward conditioning problem where we only condition on the total accumulated production for each well. After that, we can introduce additional data points or changes to the parametrization and rerun the history matching experiment if there is a need to improve the results.

We also noticed that the convergence of ESMDA is an issue since it is unclear how many steps are needed and what the optimal step lengths should be. We need more research 
on this topic. For now, we notice that IES converged in 15 iterations and with 16 ESMDA steps the variance in the ESMDA results are a little higher than in the IES results. See [14] for a discussion on the derivation and the convergence properties of the iterative smoothers.

Acknowledgements Geir Evensen was supported by a research project funded by Statoil and for the finalization of the manuscript by the Research Council of Norway through the DIGIRES project with industry partners Statoil Petroleum AS, Eni Norge AS, VNG Norge AS, DEA Norge AS, Aker BP AS, and Petrobras.

Kjersti Solberg Eikrem acknowledges the Research Council of Norway and the industry partners; ConocoPhillips Skandinavia AS, Aker BP ASA, Eni Norge AS, Maersk Oil Norway AS, DONG Energy A/S, Denmark, Statoil Petroleum AS, ENGIE E\&P NORGE AS, Lundin Norway AS, Halliburton AS, Schlumberger Norge AS, Wintershall Norge AS of The National IOR Centre of Norway for support.

The study received access to Eclipse licenses from Schlumberger and benefited from the interaction and collaborations with the Nordforsk Nordic center of excellence in data assimilation, EMBLA. We are grateful to Joakim Hove and Statoil for making their Ensemble Reservoir Tool (ERT) available at Github. Finally, constructive comments from two anonymous reviewers have contributed to a clearer and more in-depth presentation and discussion.

Open Access This article is distributed under the terms of the Creative Commons Attribution 4.0 International License (http:// creativecommons.org/licenses/by/4.0/), which permits unrestricted use, distribution, and reproduction in any medium, provided you give appropriate credit to the original author(s) and the source, provide a link to the Creative Commons license, and indicate if changes were made.

\section{References}

1. Aanonsen, S.I., Naevdal, G., Oliver, D.S., Reynolds, A., Valles, B.: Ensemble Kalman filter in reservoir engineering - a review. SPE J 14(3), 393-412 (2009). https://doi.org/10.21188/117274PA

2. Alturki, A., Baddourah, M., Pamuku, Y., Ravanelli, F., Hayder E.: An evaluation of assisted history matching methodologies for giant simulation models (2015). https://doi.org/10.2118/ 172796-MS

3. Bennett, A.F.: Inverse methods in physical oceanography. Cambridge University Press, Cambridge (1992)

4. Chen, Y., Oliver, D.S.: Ensemble randomized maximum likelihood method as an iterative ensemble smoother. Math. Geosci. 44, 1-26 (2012)

5. Chen, Y., Oliver, D.S.: Levenberg-Marquardt forms of the iterative ensemble smoother for efficient history matching and uncertainty quantification. Computat. Geosci. 17, 689-703 (2013)

6. Chen, Y., Oliver, D.S.: History matching of the Norne full-field model using an iterative ensemble smoother. SPE Reserv. Eval. Eng. 17(2), 244-256 (2014)

7. Eikrem, K.S., Nævdal, G., Jakobsen, M., Chen, Y.: Bayesian estimation of reservoir properties-effects of uncertainty quantification of $4 \mathrm{~d}$ seismic data. Comput. Geosci. 20(6), 1211-1229 (2016)

8. Emerick, A.A., Reynolds, A.C.: Ensemble smoother with multiple data assimilation. Comput. Geosci. 55, 3-15 (2013)

9. Evensen, G.: Sequential data assimilation with a nonlinear quasigeostrophic model using Monte Carlo methods to forecast error statistics. J. Geophys. Res. 99(C5), 10,143-10,162 (1994)
10. Evensen, G.: The ensemble Kalman filter: theoretical formulation and practical implementation. Ocean Dyn. 53, 343-367 (2003)

11. Evensen, G.: Sampling strategies and square root analysis schemes for the EnKF. Ocean Dyn. 54, 539-560 (2004)

12. Evensen, G. Data assimilation: The ensemble Kalman filter, 2nd edn. Springer, Berlin (2009)

13. Evensen, G.: The ensemble Kalman filter for combined state and parameter estimation. IEEE Control. Syst. Mag. 29(3), 83-104 (2009)

14. Evensen, G.: Analysis of iterative ensemble smoothers for solving inverse problems. Computat Geosci (2018). https://doi.org/10.1007/ s10596-018-9731-y

15. Evensen, G., van Leeuwen, P.J.: An ensemble Kalman smoother for nonlinear dynamics. Mon. Weather Rev. 128, 1852-1867 (2000)

16. Hanea, R., Evensen, G., Hustoft, L., Ek, T., Chitu, A., Wilschut, F.: Reservoir management under geological uncertainty using Fast Model Update. 173305-MS SPE Conference Paper (2015)

17. Houtekamer, P.L., Zhang, F.: Review of the ensemble Kalman filter for atmospheric data assimilation. Mon. Weather Rev. 144, 4489-4533 (2016)

18. Iglesias, M.A.: Iterative regularization for ensemble data assimilation in reservoir models. Computat. Geosci. 19(1), 177-212 (2015)

19. Iglesias, M.A.: A regularizing iterative ensemble Kalman method for PDE-constrained inverse problems. Inverse Prob. 32(2), 025,002 (2016)

20. Le, D.H., Emerick, A.A., Reynolds, A.C.: Iterative ensemble smoother as an approximate solution to a regularized minimumaverage-cost problem: theory and applications. SPE J., SPE173214-PA 21(6), 2195-2207 (2016)

21. van Leeuwen, P.J., Evensen, G.: Data assimilation and inverse methods in terms of a probabilistic formulation. Mon. Weather Rev. 124, 2898-2913 (1996)

22. Luo, X., Stordal, A.S., Lorentzen, R.J.: Iterative ensemble smoother as an approximate solution to a regularized minimumaverage-cost problem: Theory and applications. SPE J., SPE176023-PA 20(5), 962-982 (2015)

23. Ma, X., Hetz, G., Wang, X., Bi, L., Stern, D., Hoda, N.: A robust iterative ensemble smoother method for efficient history matching and uncertainty quantification (2017)

24. Miyoshi, T., Kalnay, E., Li, H.: Estimating and including observation-error correlations in data assimilation. Inverse Prob. Sci. Eng. 21, 387-398 (2013)

25. Nævdal, G., Johnsen, L.M., Aanonsen, S.I., Vefring, E.: Reservoir monitoring and continuous model updating using the ensemble Kalman filter. SPE Annual Technical Conference and Exhibition (SPE 84372) (2003)

26. Oliver, D.S., Chen, Y.: Recent progress on reservoir history matching: a review. Computat. Geosci. 15(1), 185-221 (2011). https://doi.org/10.1007/s10596-010-9194-2

27. Seiler, A., Aanonsen, S.I., Evensen, G., Rivenæs, J.: Structural surface uncertainty modelling and updating using the ensemble Kalman filter. SPE J. (SPE-125352-PA) 15(4), 1062-1076 (2010). https://doi.org/10.21188/125352-MS

28. Skjervheim, J.A., Evensen, G., Hove, J., Vabø, J.: An ensemble smoother for assisted history matching. SPE 141929 (2011)

29. Skjervheim, J.A., Hanea, R., Evensen, G.: Fast model update coupled to an ensemble based closed loop reservoir management. Petroleum Geostatistics (2015)

30. Stewart, L.M., Dance, S.L., Nichols, N.K.: Correlated observation errors in data assimilation. Int. J. Numer. Meth. Fluids 56, 15211527 (2008)

31. Tippett, M.K., Anderson, J.L., Bishop, C.H., Hamill, T.M., Whitaker, J.S.: Ensemble square-root filters. Mon. Weather Rev. 131, 1485-1490 (2003) 